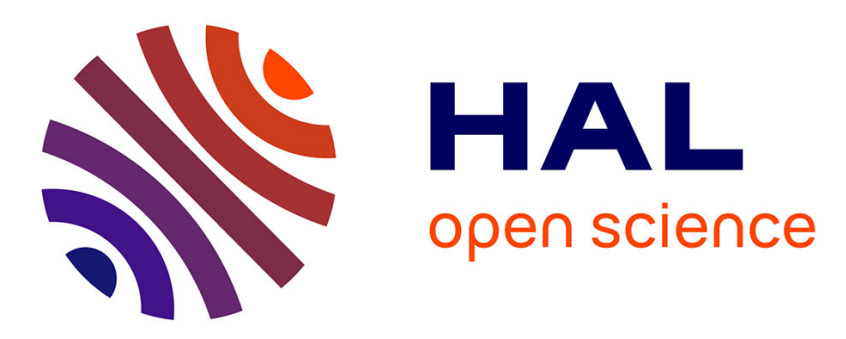

\title{
Literature Review of Deep Learning Models for Liver Vessels Reconstruction
}

\author{
Abir Affane, Marie-Ange Lebre, Utkarsh Mittal, Antoine Vacavant
}

\section{To cite this version:}

Abir Affane, Marie-Ange Lebre, Utkarsh Mittal, Antoine Vacavant. Literature Review of Deep Learning Models for Liver Vessels Reconstruction. Tenth International Conference on Image Processing Theory, Tools and Applications (IPTA), Nov 2020, Paris, France. 10.1109/IPTA50016.2020.9286639 . hal-03145758

\section{HAL Id: hal-03145758 \\ https://hal.uca.fr/hal-03145758}

Submitted on 18 Feb 2021

HAL is a multi-disciplinary open access archive for the deposit and dissemination of scientific research documents, whether they are published or not. The documents may come from teaching and research institutions in France or abroad, or from public or private research centers.
L'archive ouverte pluridisciplinaire HAL, est destinée au dépôt et à la diffusion de documents scientifiques de niveau recherche, publiés ou non, émanant des établissements d'enseignement et de recherche français ou étrangers, des laboratoires publics ou privés.

$$
\text { Copyright }
$$




\section{Literature Review of Deep Learning Models for Liver Vessels Reconstruction}

\author{
Abir AFFANE \\ Université Clermont Auvergne, \\ CNRS, SIGMA Clermont, Institut \\ Pascal, F-63000 Clermont-Ferrand, \\ France \\ Abir.affane@uca.fr
}

\author{
Marie-Ange LEBRE \\ Université Clermont Auvergne, \\ CNRS, SIGMA Clermont, Institut \\ Pascal, F-63000 \\ Clermont-Ferrand, France \\ m-ange.lebre@uca.fr
}

\author{
Utkarsh MITTAL \\ Excelerate AI, Delhi, India \\ India \\ utkarsh.mittal@excelerateai.com
}

\author{
Antoine VACAVANT \\ Université Clermont Auvergne, \\ CNRS, SIGMA Clermont, Institut \\ Pascal, F-63000 \\ Clermont-Ferrand, France \\ antoine.vacavant@uca.fr
}

\begin{abstract}
Deep learning (DL) is one of the most important machine learning methods which has achieved great success in the field of medical image analysis. DL teaches a computer model how to perform classification tasks directly from images, but since the acquisition problems of these images this method has lost its effectiveness mainly for the segmentation of complex structures such as vessels that are hardly or not visible in the raw data. Nowadays, researchers are trying to find solutions to these kinds of problem since the information of the local appearance of pixel are not enough. To illustrate the limits of using standard DL models for vessel reconstruction, we first show a comparative study based on the IRCAD dataset. This experiment motivates our study, wherein we provide a review of DL models which covers liver vessel segmentation and medical image processing, in order to confirm if these problems can be solved by $\mathrm{DL}$, and discusses a new approach to guide the experts who want to use these approaches in their work. A Systematic Literature Review (SLR) was carried out. More than $\mathbf{4 0}$ papers were founded by manual search in Elsevier, Springer and Science Direct, IEEE, 20 primary studies were finally included. According to the literature studies, we will define the most pertinent articles related to $D L$ applications for complex structure reconstruction. Results: DL based topological signature methods have better results than classical topics and DL based pixelwise.
\end{abstract}

Keywords-Deep learning, Medical image analysis, Liver vessel, Segmentation, SLR,

\section{INTRODUCTION}

Liver vessel disorders are increasing worldwide 5/10000 patients are affected, according to the "European Association of the Study of Liver" [1], These disorders collectively represent a number of diseases. The visualization and the segmentation of liver vessels is a difficult task because of their very variable, tortuous and small shapes. The evolution of computer science in research on vascular networks has revealed interest in the reconstruction and interpretation of these complex structures. Their robust extraction from biomedical images requires good acquisition during an imaging examination.

*This work was funded by the French Agence Nationale de la recherche (grant ANR-18-CE45-0018, Project R-Vessel-X http://tgi.ip.uca.fr/r-vessel-x).
The manual segmentation is a long and tedious process, and becomes impossible with a large quantity of biomedical images. The automatic extraction of this information requires a very precise segmentation, which is difficult on medical image due to their low contrast and low signal to noise ratio. Classic methods are hardly reproducible or reach a low accuracy when confronted to more complex than limited private data sets. For the segmentation of the hepatic vessels, Lesage et al. [3], published a large and relevant overview. Some interesting techniques are the 3D multiscale line filter of Sato et al. [4] n improved locally adaptive region growing algorithm from Yi \& Ra et al. [5]; Kawajiri et al. [6] proposed a thresholding segmentation approach after contrast enhancement steps, but this method will generate false positives and even false negatives.

Oliveira and al.[7] used a region growing method based on a Gaussian mixture model for the segmentation of the vessels and nodules but the bifurcations or parts of vessels were not detected, especially when the nodules or the veins are close to the edge of the liver. Recently Butdee et al. [8] proposed a 3D segmentation method by region growing, they selected two seed points, some enhancement of the gray values was released for optimal visualization, then a pixel within the hepatic vein in any slice was selected as a seed point for the $3 \mathrm{D}$ region growing algorithm to extract the hepatic vein, it was selected at the main branch of the hepatic vein.

The same process was repeated for segmentation of the portal vein to give vessels of liver at the end of processing. All these methods did not succeed in giving that the complete reconstruction of the liver vessels because they all used as information image intensities, which is generally not sufficient in MRI and CT images. Exploration of vascular images should consider the geometry and topology of vessels, the use of local appearance of the pixel is not sufficient, as illustrated by the methods we have described so far. Recently, deep learning architectures have been successfully applied to liver vessel segmentation challenges by automatically learning the hierarchies of relevant attributes directly from medical images. For example, a Convolutional Neural Network $(C N N)$ has been applied to image classification and object detection Cai et al. 
[9]. And since then, $C N N$ networks have been the most efficient networks for binary classification. ResNet, DenseNet and U-Net have been proposed in recent years with very significant classification performance.

The architecture of unsupervised DL methods is the key for experts and researchers to solve segmentation problems. Among these architectures, we find U-Net model, which has been developed in the IT department of the University of Friborg in Germany Ronneberger et al. [10] it is based on the Fully Convolutional Network $(F C N)$ and its architecture has been modified to work with fewer training images and to allow more precise segmentation. ResNet is an Artificial Neural Network $(A N N)$ that utilize skip connections, or shortcuts to jump some layers. DenseNet, $C N N, F C N$ and Graphical Convolutional Network $(G C N)$ Shin et al.[25]; Zhai et al.[3] offer original and robust developments of image analysis algorithms and artificial intelligence, integrating strong mathematical foundations, to efficiently reconstruct the vessels shape. In this paper, we present a literature review on the topic of liver vessel segmentation using DL methods.

This work employs the framework of Systematic Literature Review (SLR) in order to provide an overview of the current body of knowledge and to discuss what was proposed in the literature on the topic of liver vessel reconstruction to guide experts, engineers, researchers who want to use the DL approaches in their studies. We will explore the approaches which integrate the geometrical or topological parameters of the vessels in their networks to evaluate their performance.

The rest of the paper is organized as follows: First, we propose to illustrate the difficulty of liver vessel segmentation by DL by presenting a short comparative study based on the public IRCAD dataset, in Section 2. Then, the SLR [2], method is briefly described, and Section 3 outlines the applied research methodology to synthesize studies and expose the reviewed works that deal with the topic of liver vessel segmentation. Next in Section 4 we discuss the proposed methods, and finally, in Section 5 the conclusion of this study is expressed.

\section{Problem Statement}

To illustrate the complexity of liver vessel segmentation by using DL models, we have tested several standard architectures, trained and validated with the public IRCAD dataset. This database is composed of the 3D CT-scans, in DICOM format (with variable size $\sim 512 \times 512 \times 100$ ) of 10 women and 10 men with hepatic tumours in $75 \%$ of cases. Binary masks of the ground truth of data has been provided: Each slice is marked by expert radiologists for various target features like bone, kidney, venous system, etc. The segmented zone of interest to us is the 'venous system', which is present for 12 out of the 20 patients. This gives us a total of 1516 images and corresponding masks for liver vessels. The DL models presented in Table 1 have been implemented with Tensorflow and Keras framework, evaluated with these images by randomly selecting the data distributed into train, validation and testing sets in the ratio of $80-10-10 \%$ respectively.
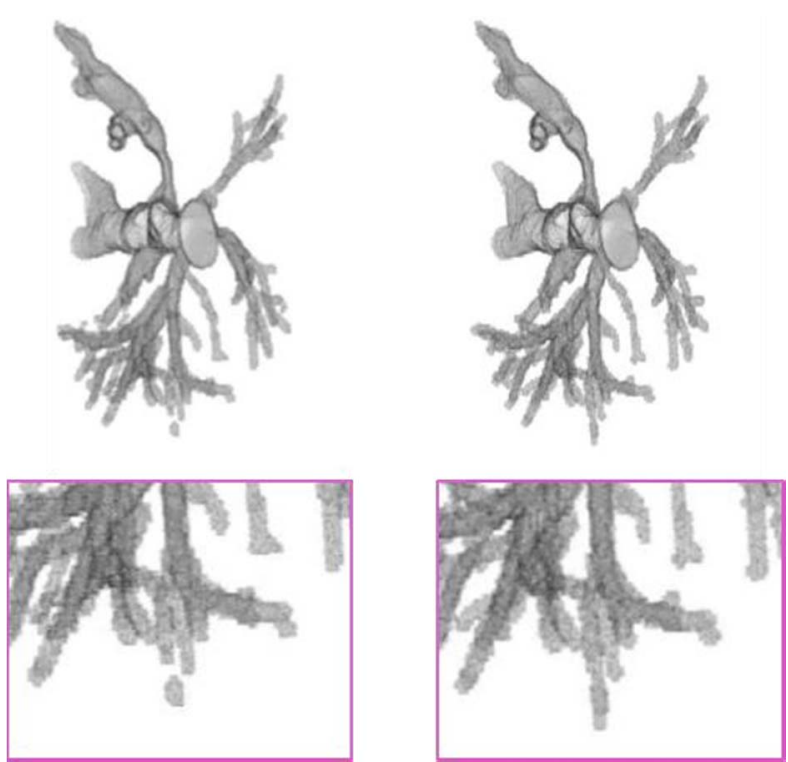

Figure 1: Segmentation by U-Net model (left) vs. ground truth (right) from IRCAD dataset, with a zoomed part (bottom).

Table 1 also presents the best Dice measure obtained for each DL model. From these experiments, we can notice that this score does not exceed $90 \%$, even if the U-Net based architecture has achieved the best value of $88 \%$, which is the best approach of the test. We have analyzed further the results obtained from this approach. Figure 1 is an illustration of a segmentation computed by the U-Net model (left), compared to the expected ground truth (right). By considering more precisely the 3D images (bottom), we can observe that the extracted vasculature has disconnections and the smallest vessels may disappear. Unfortunately, the tested DL models only consider the image intensity to learn and segment these fine and complex structure, without integrating any geometrical or topological features in their decision. This experiment has largely motivated our current research and the review presented below.

Table 1: Numerical evaluation of DL models trained and validated with IRCAD dataset.

\begin{tabular}{|l|l|l|}
\hline DL model & Reference & $\begin{array}{l}\text { Best Dice } \\
\text { measure }\end{array}$ \\
\hline FCN & Long et al. $(\mathbf{2 0 1 4 )}$ & 0.72 \\
\hline U-Net & Ronneberger et al. (2015) & 0.88 \\
\hline SegNet & $\begin{array}{l}\text { Badrinarayanan } \text { et } \text { al. } \\
(\mathbf{2 0 1 7 )}\end{array}$ & 0.67 \\
\hline PSPNet & Zhao et al. (2017) & 0.75 \\
\hline
\end{tabular}

\section{Methodology}

In this paper we apply the SLR methodology on the topic of liver vessel reconstruction using DL, the aim of SLR is to collect and analyse list of all studies that are related and to reviewing the recent blood vessel segmentation algorithms.

The SLR process is based on 3 phases: planning phase, conducting phase and reporting phase. In this section, we will focus on the planning phase to define our research objectives. 


\section{III.1. Planning the review}

In this phase we introduce foundation of this review by defining the SLR research questions.

\section{III.1.1. SLR Research Questions RQ}

The segmentation of hepatic vessel with machine learning approach is a difficult problem, the SLR (RQ) that we try to answer in this paper is as follows:

RQ1: Did the proposed methods used geometrical or topological machine learning approaches?

RQ2: Did the proposed works used DL architectures?

RQ3: What are sources of medical data?

RQ4: Did the proposed framework used to segment vessels?

RQ5: Did the proposed framework used to segment liver vessels or other blood vessels?

RQ6: What is the modality used for data acquisition?

RQ7: Have the authors implemented and validated their methods?

RQ8: Were the approach tested on complete 3D volumes or on 2D slices?

\section{III.1.2 Search methods}

Papers were founded by manual search in scientific databases. In this paper we have selected five databases to perform the SLR search process and select the adequate works as follows:

- Elsevier (https://www.elsevier.com/fr-fr)

- IEEE (https://www.ieee.org/)

- Springer (www.springerlink.com)

- Science Direct (www.sciencedirect.com)

- Google Scholar (www.scholar.google.com)

We used these following terms "Liver vessel segmentation", "DL for vessel reconstruction", and "hepatic vessel segmentation by machine learning approaches" to find suitable on DL approaches for vessel reconstruction and segmentation.

\section{III.2. Review Conduction}

III.2.1 Inclusion/Exclusion Criteria and study selection

This paper analyses more than 20 articles focused on vessel blood segmentation approaches. See Table 2. The inclusion and exclusion criteria used in this study are presented below. All the articles we chose are relevant to the domain of liver vessel segmentation. Inclusion and exclusion criteria are:

Exclusion Criteria

- Articles not written in english.

- Studies outside the domain of DL based segmentation.

- Studies outside the domain of vessel segmentation.

- Duplicate articles of the same study.

Inclusion Criteria

- Recent works(2016-2020).

- Approaches tested and validated.

- Articles written in English.

- Articles for vessel segmentation.
- Studies used unsupervised machine learning approaches for segmentation problems.

\section{III.3. Reporting the review}

We summary each analysed approach, in Table $\mathbf{1}$ and Table $\mathbf{2}$ below reporting DL technique used for vessel segmentation. Pros and cons of each method are presented too. We divide our analysis into 2 classes: DL for liver vessel segmentation, and DL for retinal vessel segmentation because these approaches could be extended to liver vessel extraction, all details are described in subsections below.

\section{III.3.1 DL for liver vessel reconstruction}

Different architectures of DL are developed in order to segment precisely the liver vessels on different types and modalities of medical imaging. We will expose these approaches according to image types.

\section{i. CT scan images}

In this category the researchers tested their methods on CT scan data. Zeng et al. [14] proposed solution for liver vessel segmentation based on extreme learning machine ELM tested on 3D CT scan images. They used an anisotropic filter to remove noise and to preserve vessel boundaries. Then, based on the knowledge vessel shapes, 3 filters are introduced: Sato, Frangi and offset medialness filters to extract features of the vessel. Then the ELM is applied to extract liver vessels voxels from background voxels. Kitrungrotsakul et al. [15] proposed Multi Deep Convolution Network for robust hepatic vessel extraction from 2D and 3D (CT) images. It consists of three deep convolution neural networks to extract features from (coronal, sagittal and transversal) planes of CT data. The three networks have shared features at the first convolution layer, but will separately learn their own features on the second one. All three networks will join again at the top layer. Ibragimov et al. [16] proposed to combine $D L$ with anatomical analysis for automatic segmentation of the liver portal vein. They applied $C N N$ to learn the consistent appearance patterns of the portal veins using a training set with reference annotations.

Yu et al. [18] presented a method of 3D Residual U-Net for liver vessel segmentation on 3D CT scan images. They introduced a residual block structure in the ResNet into the 3D U-Net, and construct a new 3D Residual U-Net architecture to segment the hepatic and portal veins from abdominal CT volumes. To deal with the challenge of pixel imbalance they developed a weighted Dice loss function, which allows vessel boundary segmentation and small vessel detection.

\section{ii. Other types of images}

The scientist tried also to segment hepatic vessel in images acquired by different modalities, but there weren't many articles because they are generally less used in the clinical analysis and they are more complicated to explore. Thomson et al. [19] introduced a reduced filter 3D U-Net for hepatic vessel reconstruction in $3 \mathrm{D}$ ultrasound (US) images proposed to achieve accurate vessel segmentation in true $3 \mathrm{D}$ and stuck $2 \mathrm{D}$ 
US images. Recently Kitrungrotsakul et al. [17] proposed an automatic vessel segmentation approach that uses a multipathways DL network. The proposed framework learns a deep network for binary classification based on extracted training patches in 3 planes (sagittal, coronal, and transverse planes) of MRI scan images. It provides a good recognition performance on 3D volumes. Their solution, VesselNet, products a vessel probability map for voxels to generate the final segmentation of liver blood vessels using a multi-scale method. Their results obtained on IRCAD data can absolutely confirm that the solution improves previous works presented in the literature.

\section{III.3.2 DL for retinal vessel segmentation}

Many DL methods have been introduced to reconstruct retinal vessel on 2D images. Fu et al. [20] who utilized the CNNs in combination with Conditional Random Field (CRF) to generate a vessel probability map. They developed this map that separates the vessels the background even in the inadequate contrast regions, and is not sensitive to the presence of tumours. They tested their method on 2D DRIVE and STAR public data. Oliveira et al. [21] proposed Fully Convolutional Neural Networks combined the multiscale analysis provided by the wavelet transform with a multiscale $\mathrm{CNN}$ to deal with the varying width and direction of the vessel structure in the retina. Their approach overtakes the results obtained by $\mathrm{Fu}$ et al. [20] Liskowski et al.[22] who proposed a supervised segmentation technique that uses a deep neural network tested on DRIVE and STAR public data sets their method solve the problem of the central vessel reflex. Laibacher et al. [23] presented a novel neural network architecture, called M2U-Net. This is a new encoder-decoder architecture that is inspired from the U-Net. It introduces pre-trained components in the encoder and contractive blocks in the decoder that are combined with bilinear up-sampling, the model was tested on the 2D DRIVE data set.

All existing methods using CNNs for retinal vessel segmentation are mainly based on local appearances learned on the image patches, without taking into account the graphic structure and the shape of the vessels. Shin et al. [25] proposed a new method of vessel segmentation based on the DL, which focuses on what is missing in the literature. They integrate a convolution network of graphs in an unified CNN architecture, where the final segmentation is deduced by combining the different types of features. It can be applied to extend any type of CNN-based vessel segmentation method in order to improve performance. Their method has been tested on angiographic images of the retina and since it takes into account a priori information on the shape of the vessels, it could improve the limits of the segmentation methods, especially at the bifurcation and in the smallest branches. All existing methods using CNNs for retinal vessel segmentation are mainly based on local appearances learned on the image patches, without taking into account the graphic structure and the shape of the vessels. Shin et al. [25] method can be applied to extend any type of CNNbased vessel segmentation method in order to improve performance.

\section{RESULTS AND DISCUSSION}

In this part we will present a summary of the selected studies and discuss the results according to our research questions described above. We have identified in Table 2 the most useful articles for our study. All the selected studies response at least on 3 RQs and most works use public data set to validate their DL model. The study and survey shows that the application of DL techniques for liver vessel extraction is still immature but still better in methods based topology. Regarding the quality of the chosen studies, since in the selection strategy, we have required the methods tested and validated with high accuracy, we assume that the picked studies are with high quality (see "Precision" in Table 2).

All the studies work well with the topic of vascular structures extraction, we will start with articles based retinal vessel segmentation that are presented in Table 2. Oleivera et al. [21] evaluated their FCN architecture on three public databases (DRIVE, STARE, and CHASE DB1) with average accuracy of $0.9576,0.9694,0.9653$. The advantage of FCN-based methods is that the network can take a raw image as input and perform fast and precise segmentation. They give promising results for the segmentation of large organs (liver, spleen, ...), but unfortunately it is hard to apply them effectively to the extraction of the vessels especially the hepatic vessels because of their very complicated shape. Fu et al. [20] developed DeepVessel framework that combines the $\mathrm{CNN}$ and Conditional Random Field (CRF) layers into an integrated deep network their method tested and validated on the DRIVE, STARE, and, CHASE DB1 data sets with an efficient running time and 0,94 accuracy of classification. The main advantage of their approach is that it is not sensible of tumors, this architecture seems to be very effective for 2D segmentation, but it is pity that it's not answered RQ1 and RQ8 and don't consider the shape and topology of vessels which will complicate the reuse of the method for application on hepatic vessels. Laibacher et al. [23] tested and validated their method on DRIVE dataset (20 training and 20 test images) and CHASEDB1 (8 training and 20 for test images).

Their approach gave good results compared with Unet, same as Liskowski et al. [22] their method solves the problem of the central vessel reflex with a good detection of fine vessels on 2D images public data sets (sensitivity $>0.87$ ) and it is not sensible on pathological cases. These two methods above also need to be enhanced to work on 3D images (RQ8) and integrate some topological information (RQ3). Because 2D approaches are unnecessary for the segmentation of the hepatic vessels and they will fail definitely because the direction of the vessel is not only in $\mathrm{X}$ and $\mathrm{Y}$ plane but also in $\mathrm{Z}$ direction, 2D methods lose important information of shape and topology of these structures, However Shin et al. [25] tried to limit this kind of problems by introducing integrate a convolution network of graphs in an unified CNN architecture. Their method tested on angiographic images of the retina and also on STARE, DRIVE, CHASE DB1 public data sets. We can clearly see improved performance for both data sets: for the CA-XRA dataset, the method scored an Accuracy, Precision of 0.915. While this method uses information's of the vessel shape and improve the 
limits of the segmentation methods especially at bifurcations and in small branches, it still needs to be improved on $3 \mathrm{D}$ images.

Studies validated on the segmentation of the liver vessels are also presented in the Table 2, The method of Zeng et al. [14] tested on six clinical CT data sets and segmented effectively liver vessels from abdominal CT images. It achieved good accuracy, sensitivity and specificity; but it may suffer from the imbalance pixel problem which causes the appearance of true positives and true negatives. Kitrungrotsakul et al. [15] used $12 \mathrm{CT}$ volumes which training data are randomly generate from $5 \mathrm{CT}$ volumes and 7 were used for testing. Their model can provide an average dice coefficient 0.830 , compared to $3 \mathrm{D}$ deep convolution neural network that provides around 0.7 and multiscale that provides 0.6 . In their recent work Kitrungrotsakul et al. [17] with a DL architecture on 3D images they tested and validated their model on 20 public data sets.

The outcome were impressive in performance in comparison with current works. This two last studies provides good results because they can be used for $3 \mathrm{D}$ images and also they are developed to deal with complex vascular structure segmentation problems. Yu et al. [18] and Thomson et al. [19] proposed 3D residual Unet tested on $C T$ images and 3D Unet tested on 3D US images respectively, for hepatic vessel segmentation both methods gives poor response on small branches and bifurcation and also have a problem of misclassification of pixels. From all these results we can deduce that the best methods are the ones that used the information of the shape and the topology of vessels as Shin et al. [25] and Kitrungotsakul et al. [15-18] but still need to improve their methods. They can be used to segment hepatic vessel because they answer the most to our requirement and research questions, particularly in term of the use of shape and geometrical parameters which allows an accurate analysis of complex structures.

Finally, we can deduce also that all these methods do not enable a robust segmentation $100 \%$, according to the dice values in Table 2. this can be due to the very low number of images for the training, outcomes will be absolutely better if the method was tested on a large quantity of data. Methods based on the topology and shape of the complex structures gave better results compared to classical ones even with less data, but still need to improve response in bifurcations and small branches.

\section{CONCLUSION}

The aim of this paper was to release an SLR on DL for liver vessel segmentation. Our goals were to investigate and identify the DL methods which reconstruct the complex structure precisely. Results reveal that some of them need to be considered in order to enhance the liver vessel extraction. These methods are based on the shape and topology structure of the hepatic vessels.

DL for vessel extraction is currently an active research area since segmentation of these structures is becoming more mature, it is time to face the different challenges that will allow us to enhance it and make it more effective. The main purposes of this literature review are to present the existed liver vessel segmentation approaches and to classify them and according to this classification we will develop a framework for liver vessel extraction. These studies allowed us to compare the different DL architectures for the segmentation of the vessels and compare their performance; we noticed that the topologicalDL methods based on the shape and the vascular geometry are the most pertinent for the reconstruction of the liver vessels. Their results will help us to set our approach based on topological parameters.

\section{REFERENCES}

[1] European association for the study of liver "EASL clinical practice guidelines: vascular disease of the liver"

[2] Roy F. Baumeiste, Marc.R Leary "Writing narrative litterature reviews" Review of General Psychology 1997, Vol. 1, No. 3, 311320

[3] Lasage D. Angelini E. Bloch I. Gareth F "A review of 3D vessel lumen segmentation techniques: models and features and extraction schemes " December 2009 in Medical image analysis 13(6):819-845

[4] Y. Sato. S Nakajima. H Atsumi. T Koller "3D multi-scale line filter for segmentation and visualizationof curvilinear structures in medicale images.” In CVRMed- MRCAS'97, 213-222, 1997.

[5] Yi J. Ra J. “ A locally adaptative region growing algorithm for vascular segmentation". 2003 in International Journal of Imaging Systems and Technology, 13(4),208-214.

[6] Kawajiri S. Zhou X. Zhang X, Hara T "Automatic segmentation of hepatic vessels in non-contrast X-Ray CT images" July 2008 in Radiological Physics and Technology 1(2):214-22.

[7] D Oliveira. R Feitosa. M Correia "Segmentation of liver, its vessels and lesions from CT images for surgical planning" BioMedical Engineering OnLine 2011

[8] C Butdee. Charnchai P. N Tanpowpong "3D plane cuts and cubic Beizier curve for CT liver volume segmentation according to Couinaud's classification" in Songklanakarin J. Sci. Technol.39 (6), 793-801, Nov. - Dec. 2017

[9] Z Cai, Q Fan, R S. Feris, N Vasconcelos “ A unified multi-scale Deep Convolutional Neural Network for fast object detection" arXiv.org > cs > arXiv: $1607.07155 \mathrm{v} 1,25$ July 2016

[10] O Ronneberger. P Fischer. T Brox "U-Net: Convolutional Networks for biomedical image segmentation" MICCAI 2015

[11] C Wang. Z Zhao. Q Ren. Y Xu. Y Yu. "Dense U-Net based on patch-based Learning for retinal vessel segmentation" 2014 in Entropy 2019, 21, 168; doi:10.3390/e21020168

[12] K He. X Zhang. S Ren. J Sun "Deep Residual Learning for image recognition" in 2016 IEEE (CVPR) 1063-6919

[13] Peng L. Chen YW. Lin L. Hu H. Li H. Chen Q. Ling X. Wang D. Han X. Iwamoto Y. "Classification and quantification of Emphysema using a multi-scale Residual Network" in IEEE J Biomed Health Inform. 2019 Nov;23(6):2526-2536.

[14] Y Zeng. Y Zhao. M Liao .B Zou. X Wangc. W Wang "Liver vessel segmentation based on Extreme Learning Machine" in Physica Medica 32 (2016) 709-716

[15] T Kitrungrotsakul. X Han. Y Iwamoto. A Foruzan. L Lin. Y Chen."Robust hepatic vessel segmentation using multi Deep Convolutional Network" in Medical Imaging 2017: Proc. of SPIE Vol. 10137, 1013711 (2017).

[16] B Ibragimov. D Toesca. D Chang. A Koong. L Xing. "Combining Deep Learning with anatomical analysis for segmentation of the portal vein for liver SBRT planning" in Phys. Med. Biol. 62 (2017) 8943-8958. 
Table 2 : Comparison of the reviewed vessel segmentation methods, CTA: Computer Tomography Angiography. AP : average precisions

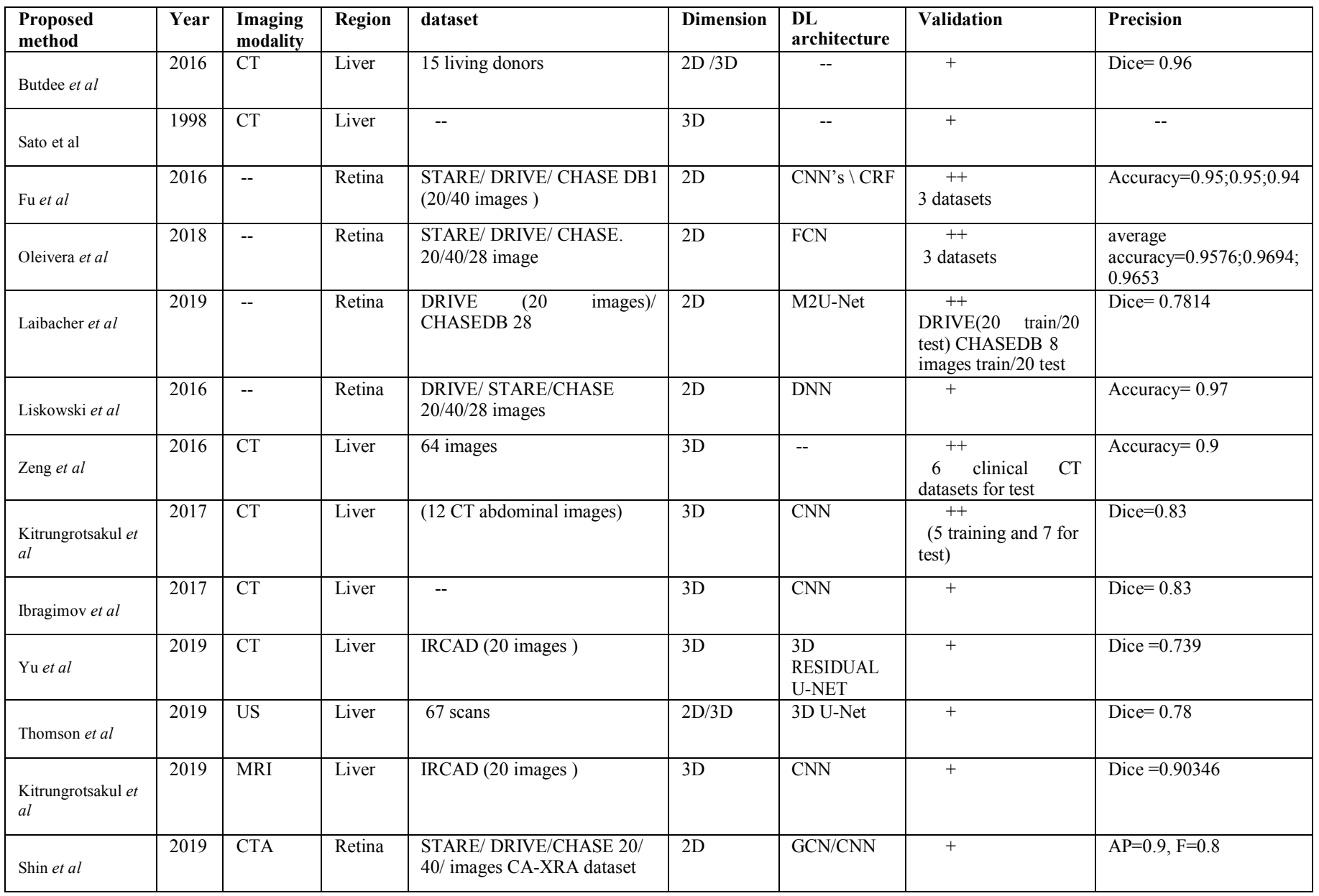

[17] T Kitrungrotsakul. X Ha. Y Iwamoto. L Lin. A Foruzan. W Xiong. Y Chen. "VESSELNET: A Deep Convolutional Neural Network with multi pathways for robust hepatic vessel segmentation" in Computerized Medical Imaging and Graphics 75 (2019) 74-83

[18] W Yu. B Fang. Y Liu. M Gao. S Zheng. Y Wang "Liver vessels segmentation based on 3D Residual U-Net" in ICIP, 978-15386-6249-6/19, 2019 IEEE

[19] B Thomson. J Nijkamp. O Ivashchenko. F Heijden. J Smit. N Kok. K Kuhlmann. T Ruers. M Fusaglia "Hepatic vessel segmentation using a reduced filter 3D U-Net in Ultrasound imaging" in MIDL 2019 Medical Imaging with Deep Learning

[20] H Fu. Y Xu. S Lin. D W K Wong. J Liu "DEEPVESSEL: Retinal vessel segmentation via Deep Learning and conditional Random field"

[21] A Oliveira. S Pereiraa. C Silva "Retinal vessel segmentation based on Fully Convolutional Neural Network" submitted to Expert Systems with Applications December 21,2018

[22] P Liskowski. K Krawiec. "Segmenting retinal bllod vessels with Deep Neural Networks in IEEE TRANSACTIONS On MEDICAL IMAGING, DOI 10.1109/TMI.2016.2546227, IEEE

[23] T Laibacher. T Weyde. S Jalali "M2U-NET: Effective and efficient retinal vessel segmentation for resource-Constrained environments" in 23 Apr 2019

[24] G Tetteh. V Efremov. N D. Forkert. M Schneider. J KirschkeB Weber. C Zimmer. M Piraud. B Menze'DEEPVESSELNET: Vessel segmentation, centerlines prediction, and bifurcations detection in 3D Angiographic volumes" (2018)

[25] S Shin. S Lee. D Yun. K Lee "Deep vessel segmentation by Learning Graphical Connectivity” (2019)
[26] D Mishra. S Chaudh S Manoharury. A Soin "Segmentation of vascular region in Ultrasound images: A Deep learning approach" 978-1-5386-4881-0/18/\$31.00 (C)2018 IEEE

[27] K Zhang. H Zhou ,2 D,Crookes. L Li. Y Shao. D Liu "Zebrafish Embryo vessel segmentation using a novel dual ResUnet model" Computational Intelligence and Neuroscience Volume 2019, Article ID 8214975, 14 pages

[28] S Moccia. EnMomi. S El Hadji. L Mattos "Blood vessel segmentation algorithm: Review of methods, datasets and evaluation metrics" Computer Methods and Programs in Biomedicine 158 (2018) 71-91

[29] J Long, E Shelhamer, T Darrell, "Fully Convolutional Networks for semantic segmentation", arXiv 1411.4038, 2014

[30] V Badrinarayanan, A Kendall, R Cipolla, "SegNet: A Deep Convolutional Encoder-Decoder architecture for image", IEEE Transactions on Pattern Analysis and Machine Intelligence, vol. 39 , no. 12 , pp. 2481-2495, 2017

[31] H Zhao, J Shi, X Qi, X Wang, J Jia, "Pyramid Scene Parsing Network" in IEEE CONFERENCE ON COMPUTER VISION AND PATTERN RECOGNITION" (CVPR), 2017.

[32] Z Zhai, M Staring, X Zhou, Q Xie, X Xiao, M. E Bakker, L J. Kroft, B P.F. Lelieveldt, G J.A.M. Boon, F A. Klok, and B C. Stoel "Linking Convolutional Neural Networks with graph Convolutional Networks: application in Pulmonary Artery-Vein separation" MICCAI 2019. 